\title{
Patterns for Competing Populations with Species Specific Nonlocal Coupling
}

\author{
A. Bayliss*, V. A. Volpert \\ Department of Engineering Sciences and Applied Mathematics, \\ Northwestern University, Evanston, IL 60208-3125, USA
}

\begin{abstract}
We study a reaction-diffusion population model for two competing species, $u$ and $v$, which also includes nonlocal integral terms that represent competition for resources. The integral terms have the form of a convolution of a given kernel function and the solution. They manifest the fact that consumption of resources by the species at a spatial location $x$ depends not just on the populations at point $x$ but rather on weighted averages of the populations in an interval about $x$. The kernel functions that we employ are characterized by two parameters, $\delta$, which gives a spatial scale of the nonlocality, with $\delta=0$ corresponding to the local case, and $\alpha$, a parameter associated with the extent of the asymmetry of the kernel, where $\alpha=0$ corresponds to a symmetric kernel, i.e., to a kernel that is an even function of its argument.

We consider the parameter regime where for $\delta=0$ the system admits a stable coexistence equilibrium, which is destabilized for sufficiently large $\delta$. Deeply in the instability region, the ensuing patterns involve arrays of islands, regions of nonzero population, separated by deadzones where the populations are essentially extinct. These structures are stationary if $\alpha=0$ and propagate if $\alpha \neq 0$. Unlike previous work, we study the patterns when the kernel parameters are different for the two species, focusing first on different $\delta$ with $\alpha=0$ and then on different $\alpha$ with the same $\delta$.
\end{abstract}

In the first case we numerically find stationary patterns consisting of (i) plateau regions for the more local species $(v)$, where the plateau value is essentially the $v$-component of the unstable equilibrium point, (ii) oscillatory patterns, where oscillations appear along the plateau and along the $u$-island and (iii) split patterns, where instead of an island, there is an island cluster of two or more neighbors separated by deadzones or near deadzones.

In the second case we consider weakly coupled systems and show that there are two kinds of patterns, (i) bound waves, when each species propagates with the same speed and (ii) unbound patterns where each species propagates with its own speed, interacting with the other species when they intersect, i.e., when the two species occupy the same or closely spaced regions. We show that whether the patterns are bound or unbound depends on the stability of the decoupled system, i.e., the system when the coupling parameters are zero. When one of the species for the decoupled system is stable, then the ensuing patterns for weak coupling is bound, while if both species are unstable for the uncoupled systems, the resulting patterns are unbound for weak coupling.

Keywords and phrases: reaction-diffusion, competing populations, nonlocality, patterns

Mathematics Subject Classification: 35K57, 92D25, 35R09, 35B36

(C) EDP Sciences, 2015 


\section{Introduction}

In this paper we analyze a model of competing species with species specific nonlocal interactions. The model is based on the diffusive logistic model (Fisher equation) with nonlocal interactions [1] and extends the scalar model to account for two competing species. The nonlocal coupling is based on the principle that competition at a specific spatial location $x$ depends not only on the populations at point $x$ but rather on a weighted average of the population in an interval about $x$. This can be the case, e.g., due to the mobility of individual members of the species that compete for scarce resources, so that the inhibiting effect of resource depletion is nonlocal [1]. When the weights are independent of the point $x$, the nonlocality can be described by convolution integrals with specified kernel functions. (An alternative interpretation of spatial nonlocality is that of a morphological property with a certain amount of degeneracy, i.e., individuals with different morphologies can require and thus compete for the same resource [5-7]. In this context, the formation of cellular structures, due, e.g., to instabilities, represents the divergence of a monomorphic population into sub-species with differing morphologies. In this paper, however, we will relate the independent variables to space and time.)

Scalar models, together with an extension that incorporated nonlocality in time, have been studied in [1, $2]$. These models admit a single spatially homogeneous non-trivial equilibrium. Conditions for instability of this equilibrium, in terms of parameters of the problem, were determined. Additional studies of the nonlocal scalar problem were presented in $[3,4,6,8]$. Conditions for instability of homogeneous equilibria were obtained in terms of properties of the convolution kernel function. It was shown that instability required that the Fourier transform of the kernel function be negative for some band of wavenumbers. Several different kernel functions were considered and stability boundaries were determined as functions of the parameters of the kernel. The emergence of stationary patterns was studied via numerical simulations in [6] and it was shown that in the instability region, small perturbations evolved to nonlinear stationary states involving islands of nonzero population, separated by apparent deadzones where the population was essentially extinct. Propagating wave solutions were also found for asymmetric kernel functions. An asymptotic analysis in [10] showed that in the limit of vanishing diffusion the islands could converge to $\delta$-functions (propagating in the case of asymmetric kernel functions).

A system modeling the interaction of two competing species was studied in [1] where it was shown that for a specific kernel function, the coexistence solution could be destabilized by sufficiently nonlocal interactions. Nonlocal predator-prey systems were studied in [9] in the context of patterns behind propagating fronts of a predator invasion. It was shown that nonlocality could destabilize stable equilibria, leading to both stationary and time dependent patterns.

Stability for the system considered here, a direct extension of the nonlocal Fisher equation, was studied in [11] where conditions for stability and instability were determined in terms of the Fourier transform of the kernel function. Numerical simulations in the fully nonlinear regime demonstrated patterns involving arrays of islands separated by deadzones. Multiple steady states, depending on the initial conditions, were found, although for stepfunction kernels, as considered here, the structure of the patterns was robust, differing initial conditions changed only the extent of the islands and deadzones. An analysis for stepfunction kernels was developed which showed that the deadzones could be approximated by the upper portion of a cosine curve. The analysis was able to predict the amplitude and the total population of the islands in terms of the kinetic parameters of the model and agreed very well with simulations.

In [12] asymmetric kernels were considered. A linear stability analysis showed that instability now occurred via a crossing of an eigenvalue from the left half plane to the right half plane off the real axis. Numerical simulations in the nonlinear regime showed patterns involving propagating arrays of islands and deadzones. Furthermore, far into the nonlinear regime colony formation, in which a parent island would spawn a colony which would grow as the parent decayed and then continue as part of the propagating array, could occur.

\footnotetext{
*Corresponding author. E-mail: a-bayliss@northwestern.edu
} 
Two key parameters associated with the nonlocality are $\delta$, a length scale associated with the extent of the nonlocality, and $\alpha$, a measure of the extent of asymmetry of the nonlocality, with $\alpha=0$ corresponding to symmetric nonlocal interaction. In previous studies these parameters were taken to be the same for each species, in effect assuming that the weights involved in averaging the contribution of each species to interspecies and intraspecies competition are the same for each species. Such an assumption may not be valid for all biological situations as differing species may respond differently to competition. For example, two competing species might operate on very different length scales in terms of their depletion of resources. In this paper we examine the role of species specific nonlocal interactions in determining pattern formation. We show that such specificity can lead to patterns very different from those obtained when the nonlocality parameters are species independent.

In section 2 we describe the model. In section 3 we describe the effect of species specific spatial scales (i.e., different values of $\delta$ for each species) on the resulting patterns. In section 4 we consider the effect of different degrees of asymmetry. In section 5 we discuss and summarize our findings.

\section{Model}

Let $u$ and $v$ represent the populations of the competing species and $t$ and $x$ denote time and space, respectively. The coupled Fisher model $[11,12]$ is

$$
\begin{aligned}
& u_{t}=d_{1} u_{x x}+c_{1} u-u\left(a_{1} \phi_{\delta_{u}, \alpha_{u}} * u+b_{1} \phi_{\delta_{v}, \alpha_{v}} * v\right), \\
& v_{t}=d_{2} v_{x x}+c_{2} v-v\left(a_{2} \phi_{\delta_{v}, \alpha_{v}} * v+b_{2} \phi_{\delta_{u}, \alpha_{u}} * u\right),
\end{aligned}
$$

where $u_{t}$ denotes the partial derivative of $u$ with respect to $t$ and similarly for other variables and $\phi * w$ denotes the convolution of two functions $\phi$ and $w$. The weighting of the nonlocality is represented by the kernel functions $\phi_{\delta, \alpha}$ where $\delta$ represents a length scale associated with the nonlocality and $\alpha$ represents the degree of asymmetry in the weighting, with $\alpha=0$ corresponding to symmetric weighting. The notation in (2.1) is designed to explicitly indicate that we now consider the case where these two parameters are species specific rather than common for both species.

The terms in (2.1) represent (i) diffusion $\left(d_{1}, d_{2}\right)$, (ii) natural growth $\left(c_{1}, c_{2}\right)$, (iii) intraspecies competition $\left(a_{1}, a_{2}\right)$ and (iv) interspecies competition $\left(b_{1}, b_{2}\right)$ and it is only via the interspecies competition that the two species are coupled. We assume that

$$
a_{1}>0, a_{2}>0, b_{1} \geq 0, b_{2} \geq 0, c_{1}>0, c_{2}>0 .
$$

We note that by suitable rescaling of $x, t, u$ and $v$, it is possible to set $d_{1}=c_{1}=a_{1}=a_{2}=1$ (among other possibilities) in (2.1).

In this paper we consider two kernel functions, (i) an asymmetric stepfunction,

$$
\phi_{\delta, \alpha}(y)= \begin{cases}\frac{1}{2 \delta}(1-\alpha), & -\delta<y<0 \\ \frac{1}{2 \delta}(1+\alpha), & 0<y<\delta \\ 0, & |y|>\delta\end{cases}
$$

and (ii) an asymmetric Gaussian,

$$
\phi_{\delta, \alpha}(y)=\frac{1}{\delta \sqrt{\pi}} \exp \left(-\left(\frac{y-\delta \alpha}{\delta}\right)^{2}\right) .
$$

For concreteness we always take the asymmetry parameter $\alpha \geq 0$, and for (2.3) we also take $\alpha \leq 1$ in order for the kernel function to be non-negative. We note that the kernel functions are scaled so that their integral over the entire real axis is unity, and they approach $\delta$-functions as $\delta \rightarrow 0$ so that in this limit the problem becomes local. 
Critical points of (2.1), i.e., spatially uniform stationary solutions $u_{0}, v_{0}$, satisfy the algebraic system of equations

$$
\begin{aligned}
& c_{1} u_{0}=u_{0}\left(a_{1} u_{0}+b_{1} v_{0}\right), \\
& c_{2} v_{0}=v_{0}\left(b_{2} u_{0}+a_{2} v_{0}\right) .
\end{aligned}
$$

It is clear from (2.5) that (2.1) admits a solution $u_{0}=v_{0}=0$ corresponding to extinction of both species. In addition, there are semi-extinction critical points,

$$
u_{0}=\frac{c_{1}}{a_{1}}, v_{0}=0, \quad u_{0}=0, v_{0}=\frac{c_{2}}{a_{2}} .
$$

Due to (2.2), these solutions are physical, i.e., the surviving species has a positive population density. The coexistence solution, where both $u_{0}$ and $v_{0}$ are nonzero, satisfies

$$
c_{1}=a_{1} u_{0}+b_{1} v_{0}, \quad c_{2}=b_{2} u_{0}+a_{2} v_{0},
$$

so that

$$
u_{0}=\frac{-b_{1} c_{2}+a_{2} c_{1}}{D}, \quad v_{0}=\frac{a_{1} c_{2}-b_{2} c_{1}}{D},
$$

where

$$
D=a_{1} a_{2}-b_{2} b_{1} .
$$

We will always assume that the parameters are such that the coexistence solution is physical, i.e., $u_{0}$ and $v_{0}$ in $(2.8)$ are both positive. Furthermore, we will assume that $\left(u_{0}, v_{0}\right)$ is stable when the coupling is local, i.e., when $\delta \rightarrow 0$. It can be easily seen that $D>0$ is necessary and sufficient for $\left(u_{0}, v_{0}\right)$ to be stable in the case of local coupling. For $\delta_{u}=\delta_{v}=\delta$ and $\alpha_{u}=\alpha_{v}=0$ (symmetric kernel functions) it was shown in [11] that for $(2.3)\left(u_{0}, v_{0}\right)$ can be destabilized for sufficiently large $\delta$ while for $(2.4)$ the coexistence critical point is unconditionally stable. In [12] it was shown that for both $(2.3)$ and $(2.4)\left(u_{0}, v_{0}\right)$ can be destabilized for sufficiently large $\delta$ for $\alpha>0$. For $\alpha=0$ destabilization occurs via an eigenvalue of the linearized system crossing from the left half plane to the right half plane along the real axis. Thus, stationary patterns ensue as a result of the instability. In contrast, for $\alpha>0$ destabilization occurs via a nonreal eigenvalue crossing the imaginary axis with nonzero wave number and propagating patterns appear. Furthermore, it was shown that as $\delta$ increased from the stability boundary the spatial patterns evolved to arrays of islands, regions of nonzero population, separated by deadzones, regions where the populations were essentially extinct. These arrays were stationary for $\alpha=0$ and propagating for $\alpha>0$. In this paper we numerically study patterns associated with species specific parameters, i.e., $\delta_{u} \neq \delta_{v}$ and $\alpha_{u} \neq \alpha_{v}$.

Our numerical method to compute patterns consists in solving (2.1) subject to specified initial conditions and periodicity on the interval $[-L, L]$. We employ a semi-implicit Fourier pseudo-spectral scheme where nonlinear terms are evaluated in physical space and then transformed to Fourier space for temporal updates [11]. We integrate in time until steady state conditions are achieved.

\section{Patterns for symmetric nonlocal interactions}

In this section we consider (2.1) with the kernel function (2.3) and with symmetric nonlocal coupling, i.e., $\alpha_{u}=\alpha_{v}=0$. (We do not consider $(2.4)$ here since $\left(u_{0}, v_{0}\right)$ is unconditionally stable for $\alpha=0$.) Under these conditions steady state implies stationary patterns and for all computations presented here we integrate until numerical approximations to the time derivatives of the solution get sufficiently small, specifically, until $\max \left(\left|u_{t}\right|,\left|v_{t}\right|\right)<10^{-10}$, where the maximum is taken over all spatial points.

We take the parameters

$$
d_{1}=d_{2}=2, c_{1}=49, c_{2}=9, a_{1}=250, a_{2}=30, b_{1}=100, b_{2}=40, L=1000,
$$


which are the same as those employed in [11]. We consider $\delta_{u}=\delta=175$ (the same value as was considered in [11]) and take $\delta_{v}=\delta / C$ where $C \geq 1$. Thus, in the nonlocal competition term species $v$ operates over a smaller spatial interval than species $u$, considerably so if $C$ is large. If $C \rightarrow \infty$ then the coupling terms involving species $v$ become local. For many computations we employ an 8-mode perturbation of the coexistence critical point as the initial condition,

$$
u(x, 0)=u_{0}+\varepsilon \sin (8 \pi x / L), \quad v(x, 0)=v_{0}+\varepsilon \sin (8 \pi x / L),
$$

where $\varepsilon=.01$. Other computations involved continuation, where the initial condition was the steady state result from a different value of $C$. Continuation can lead to different steady states than for the initial condition (3.2) and we categorize these below. All computed steady state patterns involved an 8-fold replication of an island/deadzone pattern, except that the islands now had structure to them and it is probably more illuminating to describe the pattern as an 8-fold replication of an island/deadzone cluster. In most cases we focus on an individual cluster within this 8-fold array.

For the stepfunction kernel (2.3) all previously reported patterns with $\delta_{u}=\delta_{v}=\delta$ (i.e., $C=1$ ) involved island/deadzone arrays, where there was no oscillatory structure to the island. In fact, in [11] it was shown that the islands could be described to a very close approximation as the upper lobe of a cosine curve. When $C>1$ this behavior is no longer the case. There are three primary effects that we have found: (i) $v$-islands exhibit a plateau region where the population is essentially constant, with the constant value being close to the $v$-component of the coexistence critical point $\left(v_{0}\right)$, (ii) spatially oscillatory patterns, where oscillations appear along the island and (iii) islands split into a cluster of two neighboring islands with a deadzone or near deadzone between them.

In order to describe these new patterns we employ the following terminology and notation: (i) We call a pattern "split" $(S)$ if the $u$-profile splits into a cluster of two or more subislands with a deadzone or near deadzone between them. If no such splitting occurs we term the pattern "intact" $(I)$. This broad classification refers only to species $u$. We note that classification of a pattern as intact does not mean there cannot be structure within the island. There can be as will be seen below. The term "intact" simply means that there is no deadzone or near deadzone between different regions of the island or the island cluster. (ii) We classify each pattern by the number of relative maxima within each island. Thus, if for any particular island cluster the $v$ island has $n$ maxima and the $u$-island has $m$ maxima we refer to the island as a $V n U m$ island and call $n$ the $V$-number of the pattern and $m$ the $U$-number of the pattern. We only consider oscillations of amplitude $10^{-10}$ or greater and when we find patterns with very small oscillations we rerun the computation with a finer grid to insure that the oscillations are not the result of numerical errors. We note that if the island is sufficiently steep, there can be oscillations which do not lead to relative maxima. We do not account for such oscillations in our labeling system. We further note that there is no reason why an intact pattern cannot have a $U$-number greater than 1 (a split island must have a $U$-number greater than one). We have found that while there can be multiple steady states for the same value of $C$, in virtually all cases the different steady states have different $V$-numbers or $U$-numbers. (iii) We refer to an island as a plateau if the population is essentially constant, or equivalently the profile is essentially horizontal across the primary extent of the island.

Intact patterns occur for values of $C$ near 1. (All of the patterns that we have found for $C=1$ have been intact [11]). In Figure 1 we plot $u$ and $v$ for $C=1$ and $C=2$. For both values of $C$, there is no splitting of the $u$-islands and there are no oscillations along the island. In terms of our naming convention, both solutions would be called a $V 1 U 1$ solution. For both values of $C$ there are 8 identical islands within the computational domain separated by deadzones. The two species occupy essentially the same space. There is a phase shift between the solutions with $C=1$ and $C=2$, however, any solution is invariant with respect to translation since the coefficients are independent of $x$. The primary effect is that for $C=2$ the amplitude of the $u$-island is slightly reduced from that of the $C=1$ case while the amplitude of the $v$-island exhibits a more significant reduction. We believe that this is a consequence of the fact that the convolution for species $v$ takes in less of the neighboring deadzone when $\delta_{v}$ is reduced. 
As a consequence the competition terms involving $v$ are larger, leading to a reduction in amplitude of the islands for each species.

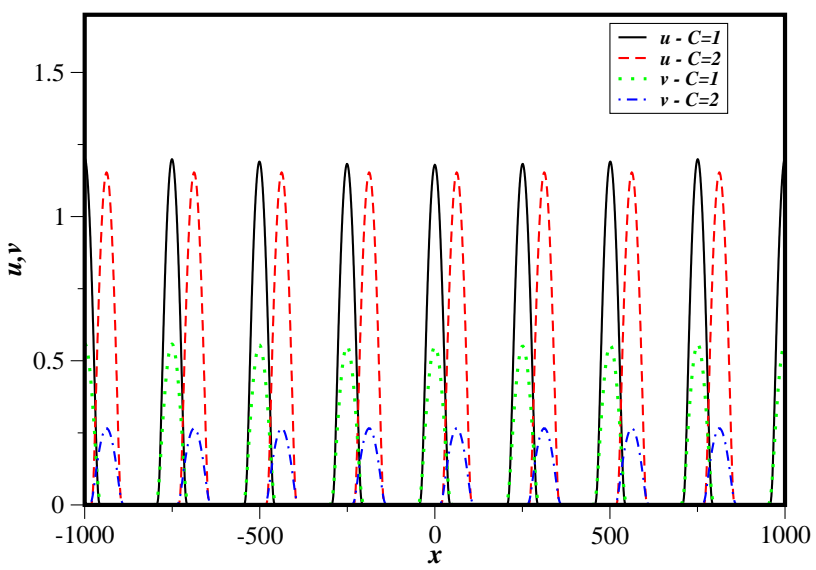

Figure 1. $u$ and $v$ with $C=1$ and $C=2$.

Intact solutions also occur for $C$ large. We illustrate such solutions in Figure 2 for $C=$ $\infty, 22.125,22.075$. There are eight identical island clusters spread over the interval $[-1000,1000]$. The figure is restricted to just one island cluster, over the interval $[100,300]$. The intact solution is prone to instabilities, as can be seen by the oscillations that develop across the island as $C$ is decreased. For $C=22.125$ we have a $U 3$ solution with small amplitude oscillations. $C=22.075$ is a $U 5$ solution with more pronounced oscillations. (All solutions have a $U$-number of 1 for $C \geq 22.25$.)

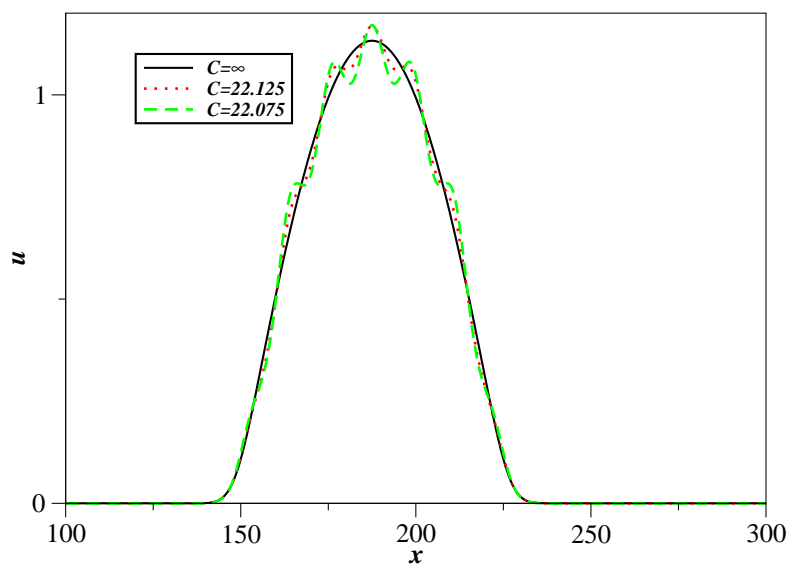

FiguRE 2. $u$ over one island cluster for $C=\infty, 22.125,22.075$. 
We next examine the structure of the $v$-islands for large $C$. In Figure 3 we plot one island structure for $C=\infty, 23,22.075$. For all three values of $C$, the $v$-island is either a plateau at a nearly constant value of $v$ or an approximate plateau, with small oscillations about a nearly constant value of $v$. This value is very close to the $v$-component of the coexistence critical point $(0.082857$, whereas the plateau height for $C=\infty$ is 0.082893$)$. The intact $u$ /plateau $v$ structure is unstable as $C$ decreases as can be seen by the oscillations that develop across the island for both $u$ and $v$. For $C=\infty$ the solutions is a $V 1 U 1$ solution, while for $C=23$ the solution is a $V 7 U 1$ solution, although only the oscillations at the edge of the plateau are visible in the figure, while for $C=22.075$ the solution is a $V 9 U 5$ solution. We note that in all cases that we have computed, the oscillations are symmetric around the center of the island for both $u$ and $v$. The appearance of solutions like $V 7 U 1$ or $V 9 U 5$, i.e., solutions with different $U-$ and $V$-numbers deserves some discussion. We remark that if these solutions appear as a result of instability of another stationary solution (say, the $V 1 U 1$ solution with a plateau in $v$ that is described above for $C=\infty$ ) then the perturbation that leads to the new steady state has the same wavenumber for both $u$ and $v$, so one might expect to see the same number of maxima in the $u$ and $v$ components. This does not necessarily happen because the perturbation is superimposed upon very different $u$ and $v$ background profiles. As a result, we may observe a different number of oscillations in the $u$ and $v$ components.

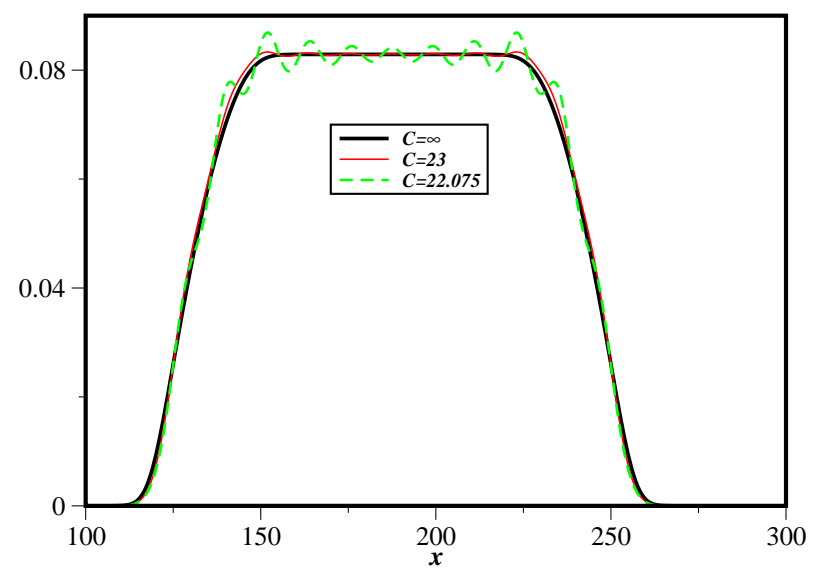

FiguRE 3. $v$ over one island cluster for $C=\infty, 23,22.075$.

In order to understand the plateau behavior for $v$, as well as the oscillations that develop as $C$ decreases from infinity, consider the case $C=\infty$ so that interactions involving species $v$ are local, while interactions involving species $u$ are nonlocal and since $\delta=175$, for any value of $x$, these interactions extend over the interval $[x-175, x+175]$.

Examination of the typical $u$-islands (see Figure 2) shows that their extent is less than $\delta$ and they are surrounded by a deadzone which is of extent $O(\delta)$. Since the kernel function is a step function, the nonlocal terms in (2.1) are independent of the spatial coordinate $x$ and are just the mean of the $u$-island, except near the edges of the island where the nonlocal terms involve a contribution from the neighboring island. (This observation is discussed in detail in [11], where it is also shown that the $u$-islands can be modeled by the upper lobe of a cosine curve, again except near the edges of the island.) Call this constant $C U$. Since we consider large $C$, the $v$-interaction will be essentially local. Therefore at steady state $v(x)$ and $u(x)$ will satisfy the system 


$$
\begin{aligned}
& 0=d_{1} u_{x x}+u(x)\left(c_{1}-a_{1} C U-b_{1} v(x)\right), \\
& 0=d_{2} v_{x x}+v(x)\left(c_{2}-a_{2} v(x)-b_{2} C U\right) .
\end{aligned}
$$

Due to the plateau behavior in $v$, we can neglect the diffusion term in (3.3b). Thus, since we are considering the region where neither population is extinct, $v(x)$ satisfies the equation

$$
c_{2}=b_{2} C U+a_{2} v(x) .
$$

If we further neglect diffusion for species $u$, which is small because the $u$-island is relatively wide, then we obtain

$$
c_{1}=a_{1} C U+b_{1} v(x) .
$$

The system of equations (3.4) and (3.5) is exactly the system (2.7) and admits the solution

$$
C U=u_{0}, v(x)=v_{0} .
$$

This analysis can also explain the onset of oscillations for the intact/plateau solutions, as a linear stability analysis of just the scalar equation

$$
v_{t}=d_{2} v_{x x}+c_{2} v-v\left(a_{2} \phi_{\delta_{v}, \alpha_{v}} * v+b_{2} u_{0}\right),
$$

where $\alpha_{v}=0$, gives a stability limit very close to the point where oscillations appear in the $u$ and $v$ profiles.

We next consider split solutions. We illustrate these solutions in Figure 4 where we plot $u$ over one island cluster for $C=20,17,7.5,6$. The solutions for $C=20$ and $C=17$ are $V 7 U 7$ solutions and while not visible in the figure there are low level oscillations between the two spikes for these solutions. In contrast, the $C=7.5$ and $C=6$ solutions are $V 4 U 2$ solutions and there are no oscillations between the two spikes in population.

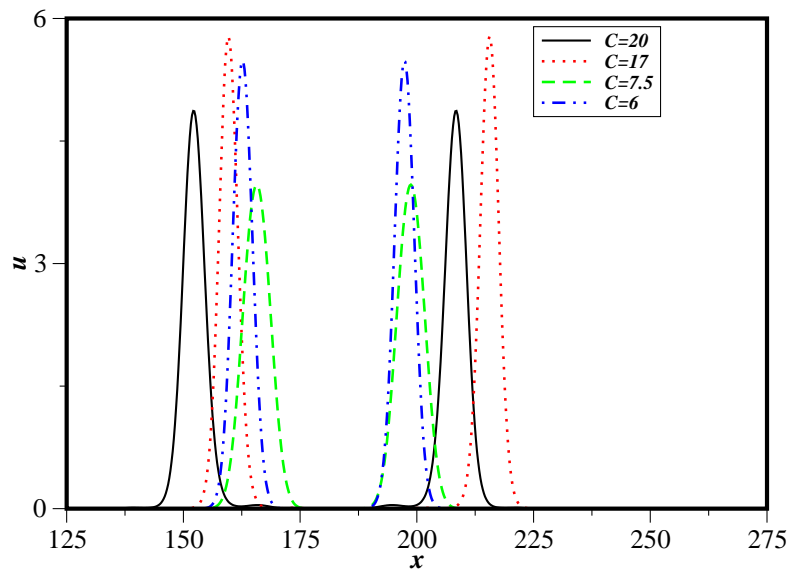

Figure 4. $u$ over one island cluster for $C=20,17,7.5,6$.

We speculate that the onset of splitting as $\delta_{v}$ increases (i.e., as $C$ decreases) arises from an increase in the $v$-competition term at the edges of the $v$-plateau. The $u$-island, resembling the upper lobe 
of a cosine curve, and the $v$-island both occupy essentially the same region of space, i.e., both species coexist over the island region. As $\delta_{v}$ increases the actual $v$-competition term at the edges of the plateau and hence of the $u$-island gets smaller, because the convolution includes a larger contribution from the deadzone adjacent to the plateau. If the competition term is reduced, this allows the population of species $u$ to increase at the edges of the island. This, in turn, increases the effective competition felt by species $u$ in the central region of the island, causing the population of species $u$ there to decrease. This in turn reduces competition further at the edge of the island, leading to a further increase in the population of species $u$. The whole process could be thought of as a self-reinforcing one, leading to a deadzone in what was the center of the $u$-island and thus a splitting of the $u$-island. For smaller values of $C$, i.e., as $\delta_{v} \rightarrow \delta$, the splitting effect cannot be sustained as in this case at the edge of each island, the nonlocal $v$-competition terms can pick up a nonzero contribution from the adjacent island cluster.

The corresponding $v$-island clusters are shown in Figure 5. For $C=20$ and $C=17$, there are 7 oscillations across each $v$-island so that the solution is a $V 7 U 7$ solution. The oscillations are roughly sinusoidal about the $v$-component of the critical point so that there is still a trace of the plateau behavior seen for larger values of $C$. For $C=7.5$ and $C=6$ there are four oscillations within each $v$-island and the solution is a $V 4 U 2$ solution. For these smaller values of $C$, the cluster is composed of sub-islands separated by near deadzones and there is no visible remnant of the plateau behavior.

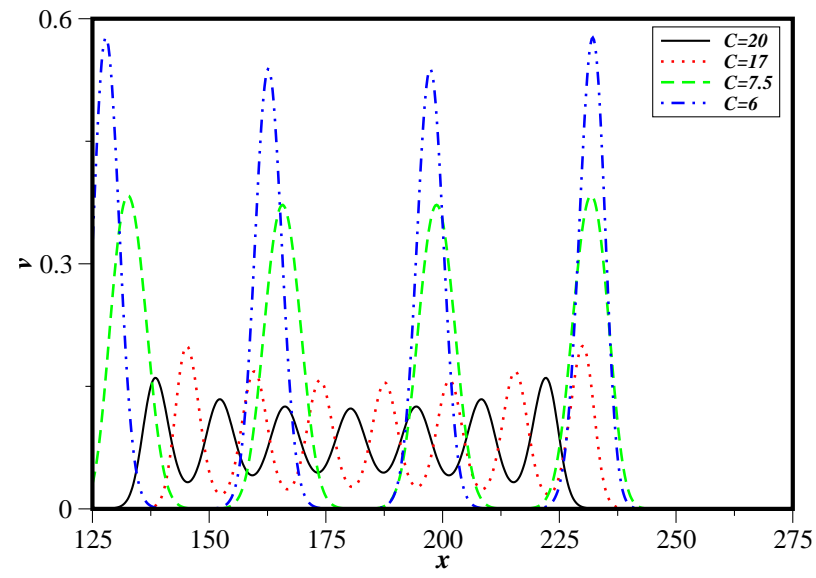

Figure 5. $v$ over one island cluster for $C=20,17,7.5,6$.

We list a sampling of computed solutions in Table 1. The table lists the $V$ and $U$ number of each solution, the maximum value of $u, u_{\max }$, as well as the initial condition employed and whether the solution is intact $(I)$ or split $(S)$.

We make the following points regarding this table:

1. We only find intact solutions for $C$ large and for $C$ around unity $\left(\delta_{v} \simeq \delta\right)$. For intermediate values of $C$ we only find split solutions.

2. The intact solutions appear to be subject to an instability as $C$ decreases.

3. There is only one case that we have found where there are two different solutions for the same $C$ with the same $v$-number $(C=21)$. For all other values of $C$ that we have investigated, for each value of $C$ there is at most one solution with a given $V$-number. Thus, we feel that this is a reasonable way to categorize computed patterns. 


\begin{tabular}{|c|c|c|c|c|c|}
\hline$C$ & $v$-number & $u$-number & $u_{\max }$ & Init & $I$ or $S$ \\
\hline$\infty$ & $V 1$ & $U 1$ & 1.13245 & 8-mode perturbation & $I$ \\
\hline 100 & $V 1$ & $U 1$ & 1.13294 & 8-mode perturbation & $I$ \\
\hline 25 & $\overline{V 8}$ & $U 1$ & 1.14013 & 8-mode perturbation & $I$ \\
\hline 24 & $V 7$ & $U 1$ & 1.14162 & 8-mode perturbation & $I$ \\
\hline 23 & $V 7$ & $U 1$ & 1.14576 & 8-mode perturbation & $I$ \\
\hline 22.5 & $V 7$ & $U 1$ & 1.15147 & 8-mode perturbation & $I$ \\
\hline 22.125 & $V 9$ & $U 3$ & 1.16816 & 8-mode perturbation & $I$ \\
\hline $22.075(A)$ & $V 9$ & $U 5$ & 1.17079 & 8-mode perturbation & $I$ \\
\hline 23 & $V 8$ & $U 4$ & 3.04708 & continuation from $B$ & $S$ \\
\hline 22.75 & $V 8$ & $\overline{U 4}$ & 3.47348 & continuation from $C$ & $S$ \\
\hline 22.5 & $V 8$ & $U 4$ & 3.73307 & continuation from $C$ & $S$ \\
\hline 22.25 & $V 8$ & $U 2$ & 3.93131 & continuation from $C$ & $S$ \\
\hline $22.075(C)$ & $V 8$ & $\overline{U 2}$ & 4.05005 & continuation from $B$ & $S$ \\
\hline $22.05(B)$ & $V 8$ & $U 2$ & 4.06559 & 8-mode perturbation & $S$ \\
\hline 22 & $V 8$ & $U 2$ & 4.09406 & 8-mode perturbation & $S$ \\
\hline 21.5 & $V 8$ & $U 4$ & 4.33160 & 8-mode perturbation & $S$ \\
\hline 21 & $V 7$ & $U 4$ & 4.47660 & continuation from $D$ & $S$ \\
\hline 20 & $V 7$ & $U 7$ & 4.86875 & 8-mode perturbation & $S$ \\
\hline 19 & $V 7$ & $U 7$ & 5.30641 & 8-mode perturbation & $S$ \\
\hline 18 & $\overline{V 7}$ & $U 7$ & 5.61392 & 8-mode perturbation & $S$ \\
\hline 17 & $V 7$ & $U 7$ & 5.78129 & 8-mode perturbation & $S$ \\
\hline 24 & $V 7$ & $U 2$ & 6.39006 & continue from $F$ & $S$ \\
\hline $23(F)$ & $V 7$ & $\overline{U 4}$ & 6.45825 & continue from $E$ & $S$ \\
\hline $22(E)$ & $V 7$ & $U 4$ & 6.50537 & continue from $D$ & $S$ \\
\hline $21(D)$ & $V 7$ & $U 4$ & 6.46526 & 8-mode perturbation & $S$ \\
\hline $20(G)$ & $V 6$ & $U 4$ & 6.32337 & continue from $D$ & $S$ \\
\hline 19 & $V 6$ & $U 6$ & 6.40576 & continue from $G$ & $S$ \\
\hline 16 & $V 6$ & $U 6$ & 5.01991 & 8-mode perturbation & $S$ \\
\hline 15 & $V 6$ & $U 6$ & 5.35931 & 8-mode perturbation & $S$ \\
\hline 10 & $V 5$ & $U 5$ & 5.44315 & 8-mode perturbation & $S$ \\
\hline 7.5 & $V 4$ & $U 2$ & 3.96541 & 8-mode perturbation & $S$ \\
\hline 7 & $V 4$ & $U 2$ & 4.32834 & 8-mode perturbation & $S$ \\
\hline 6.5 & $\overline{V 4}$ & $\overline{U 2}$ & 4.82290 & 8-mode perturbation & $S$ \\
\hline $6(H)$ & $V 4$ & $U 2$ & 5.48211 & 8-mode perturbation & $S$ \\
\hline 5.5 & $\overline{V 2}$ & $\overline{U 2}$ & 1.96602 & 8-mode perturbation & $S$ \\
\hline $5.5(I)$ & $V 4$ & $U 2$ & 6.05795 & continue from $H$ & $S$ \\
\hline 5 & $V 3$ & $U 1$ & 4.33452 & continue from $I$ & $S$ \\
\hline 5 & $\overline{V 2}$ & $U 2$ & 2.10756 & 8-mode perturbation & $S$ \\
\hline 3 & $V 2$ & $U 2$ & 4.46053 & 8-mode perturbation & $S$ \\
\hline 2 & $V 1$ & $U 1$ & 1.15276 & 8-mode perturbation & $I$ \\
\hline 1 & $V 1$ & $U 1$ & 1.19047 & 8-mode perturbation & $I$ \\
\hline
\end{tabular}

TABLE 1. Solutions obtained for a range of values of $C$.

4. Since our numerical method involves solving the initial value problem until steady state conditions are achieved, it is difficult for us to delineate specific solution branches as $C$ varies. However, grouping solutions by $V$-number and using $u_{\max }$ as a measure of the solution, appears, at least in most cases, to give a representation of solution branches and to distinguish different bistable solutions. As one illustration we plot in Figure $6 u_{\max }$ for the intact solutions for large $C$ and for the $V 8$ split solutions. (We did not include intact data for $C>25$ because $u_{\max }$ does not change by very much and we wanted to keep the horizontal scale relatively small in order to more clearly illustrate the bistability.) 


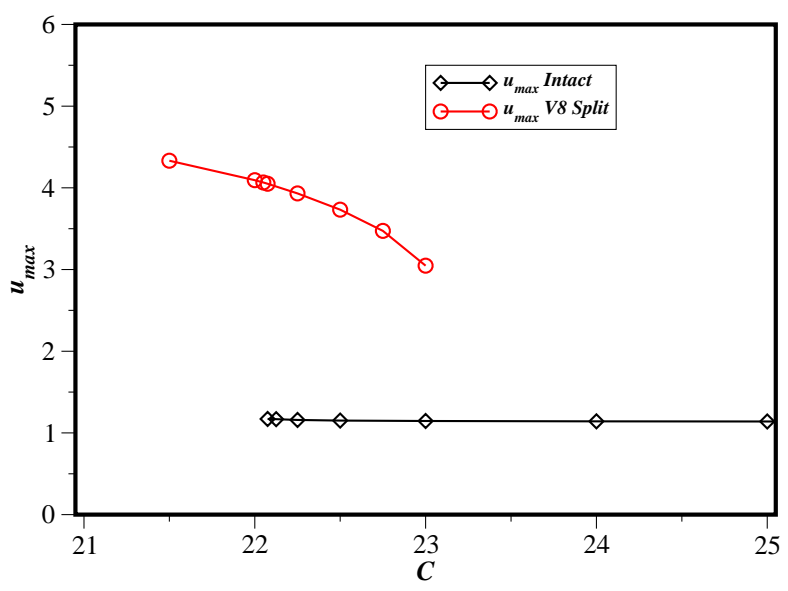

FiguRE $6 . u_{\max }$ for the intact solutions for large $C$ and the $V 8$ split solutions.

\section{Species specific asymmetry in the nonlocal coupling}

In this section we consider (2.1) with the parameters

$$
d_{1}=d_{2}=1, c_{1}=c_{2}=1, a_{1}=a_{2}=1, b_{1}=\epsilon, b_{2}=2 \epsilon, L=100, \delta_{u}=\delta_{v}=\delta,
$$

where $\epsilon$ is small so that we focus on competing species which are only weakly coupled. (As mentioned above, (2.1) can be nondimensionalized so that all parameters are unity except for $d_{2}, c_{2}$ and the coupling parameters $b_{1}$ and $b_{2}$.) We consider the behavior for both kernel functions, the asymmetric stepfunction (2.3) and the asymmetric Gaussian (2.4).

These two kernel functions illustrate two different phenomena. For the asymmetric stepfunction (2.3) the system will be unstable for sufficiently large values of $\delta$ for all values of $\alpha$, down to $\alpha=0$. Thus, if we were to consider the decoupled system $\left(b_{1}=b_{2}=0\right)$ both $u$ and $v$ would be unstable for sufficiently large $\delta$, and if $\alpha_{u}>0, \alpha_{v}=0$ then $u$ would exhibit traveling patterns while $v$ would exhibit stationary patterns. In this case it is not unreasonable to expect that for nonzero, but small, coupling parameters, the two species would not propagate together as a bound traveling wave, i.e., both species propagate with the same speed, but rather each species would propagate according to the behavior predicted for $\epsilon=0$. Thus, species $u$ would propagate at its speed, while species $v$ would propagate at a speed near zero, since for $\alpha_{v}=0$ and $\epsilon=0$ stationary patterns will occur for species $v$. The two species would only interact when two patterns intersected, i.e., at times when the two species occupied the same or closely spaced regions.

In contrast, for the Gaussian (2.4) there is unconditional stability when $\alpha=0$. Thus, if we consider the decoupled system and $\alpha_{u}>0, \alpha_{v}=0$, then while the system would in principle be unstable, $u$ would exhibit traveling patterns while for $v$ there would be no patterns. In this case it is not unreasonable to expect that $v$ would be "slaved " to $u$ for small, but nonzero coupling parameters. We show below that these behaviors are in fact what occurs.

In order to illustrate the dynamical pattern, we present contour plots in the $x-t$ plane for the two species. For clarity, we take only one contour level for each species. In the figures below we choose a contour level for each species that is below the maximum for that species rather than at the maximum. Thus, each island is represented by two adjacent curves in the $x-t$ plane with the distance between the curves a measure of how far from the maximum the chosen contour level is. For a traveling wave, 
each island would then be represented by two closely spaced parallel lines with slope the inverse of the propagation speed. (Note that when the islands are not bound together the amplitudes of the islands will not necessarily be constant in time due to species interaction.) In practice, we choose the contour levels for each case to give what we feel to be the clearest representation of the pattern.

\subsection{Asymmetric stepfunction}

For all computations in this subsection we took $\delta=40$. The initial conditions were a random perturbation of the coexistence critical point. In all cases that we computed the random initial conditions evolved to a pattern of four propagating islands for each species.

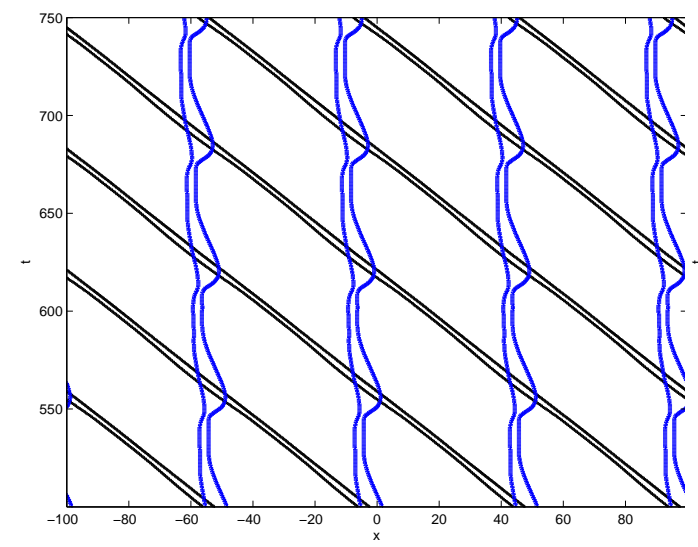

(a) $\alpha_{v}=0$

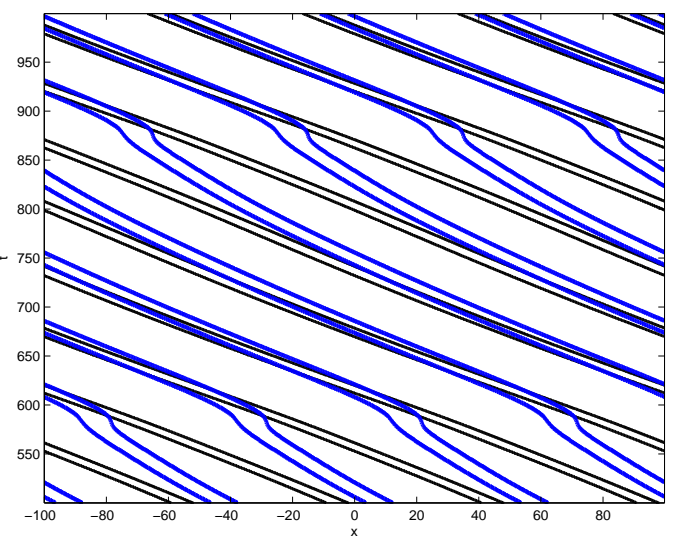

(b) $\alpha_{v}=0.4$

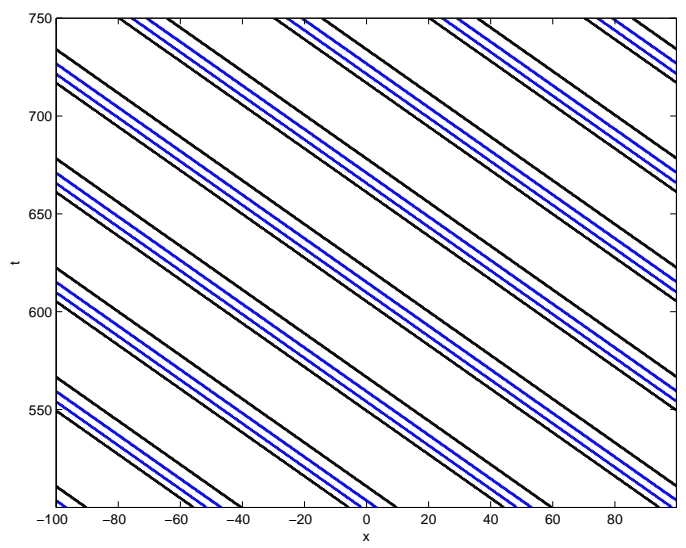

(c) $\alpha_{v}=0.5$

Figure 7. Contour plots of $u$ and $v$ with $\alpha_{u}=0.5$ and different values of $\alpha_{v}$. Other parameters are given in (4.1) with $\delta=40$ and $\epsilon=0.1$. Kernel function is (2.3). $v$-contour is in blue in online version of this paper.

Figures 7(a)-7(c) show contours of the computed patterns for $\alpha_{u}=0.5$ and $\alpha_{v}=0,0.4,0.5\left(=\alpha_{u}\right)$, respectively and for $\epsilon=0.1$. Figure 7 (a) shows that $u$ propagates essentially as a traveling wave (contours straight lines in the $x-t$ plane), while the islands for $v$ propagate nearly vertically, consistent with the conjecture that they originated from stationary patterns that would be obtained with $\epsilon=0$ (system decoupled). Said another way, it appears that when the coupling coefficients $b_{1}$ and $b_{2}$ are sufficiently 
small the weakly coupled system (2.1) is a perturbation of the decoupled system and the computed pattern is a perturbation of what would be expected from the decoupled system and therefore the two species are not bound to each other. When the patterns intersect there is some distortion in the $v$-contours, including a small retrograde motion. The effect of the interaction on the $u$ contours is not visible on the scale of the figure.

Figure 7(b) shows contours for the computed pattern with $\alpha_{u}=0.5$ and $\alpha_{v}=0.4$. The $u$-waves and $v$-waves are still unbound, but since the difference $\alpha_{u}-\alpha_{v}$ is now small, the speed of the $v$-wave is close to that of the $u$-wave (contours nearly parallel in the figure), however, the speeds are not the same and as time evolves each $v$-island disengages from its companion $u$-island and latches on to the adjacent $u$-island (or equivalently is overtaken by the adjacent $u$-island). In order to illustrate these interactions this figure is taken over a longer time interval than that for Figure 7(a).

Figure 7(c) shows contours for the computed pattern with $\alpha_{u}=0.5$ and $\alpha_{v}=0.5$. In this case the waves for species $u$ and $v$ are bound together and propagate as traveling waves.

In order to understand the nature of the interactions when the two species intersect we focus on the case $\alpha_{u}=0.5, \alpha_{v}=0.4$ shown in Figure 7(b). We note that this figure does not provide amplitude information, but rather serves only to trace out the trajectories of the $u$ - and $v$-islands. The figure indicates that the speed of the $u$-islands is roughly constant while the speed of the $v$-islands varies. Call the time corresponding to the bottom of the figure $t_{0}$ and the time corresponding to the upper part of the figure $t_{1}$. As can be seen in the figure, at $t=t_{0}$ the two species are disjoint, while at $t=t_{1}$ the two species are roughly coincident. In Figure 8 we plot $u$ and $v$ at these two times. The $x$-axis is restricted so that only one island is shown for each species and for each time.

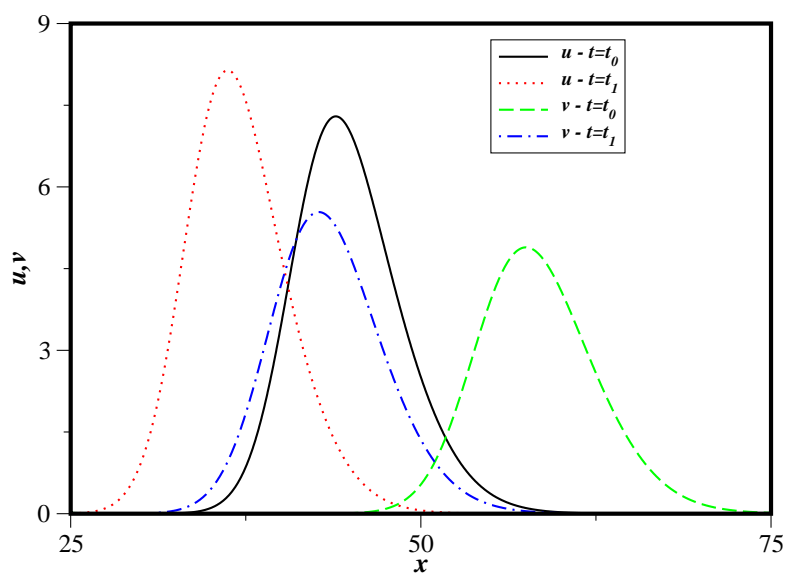

FiguRE 8. $u$ and $v$ at times $t_{0}$ and $t_{1}$ for $\alpha_{u}=0.5$ and $\alpha_{v}=0.4$.

The amplitudes for both species are larger at $t=t_{1}$ (islands roughly coincident) than at $t=t_{0}$ (islands disjoint). This is also the case for the total integrated populations within each island as can be seen from Table 2. We attribute this to the fact that when the islands are disjoint interspecies competition is greater due to the nonlocal effects of the two neighboring islands. Thus the most favorable situation for the two species is when the islands are coincident. (We note that this is not the case for a cubic (Nagumo) population model [13].) The behavior of the $u$ and $v$ contours in Figure 7(b) may be explained by this observation. If we speculate that the system evolves so that the two species "thrive", or at least 


\begin{tabular}{|c|c|c|}
\hline Species & Time & Total population of Island \\
\hline \hline$u$ & $t_{0}$ & 67.2578 \\
$u$ & $t_{1}$ & 70.6039 \\
$v$ & $t_{0}$ & 50.3131 \\
$v$ & $t_{1}$ & 54.3366 \\
\hline
\end{tabular}

TABLE 2. Total population of islands shown in Figure 8.

are as successful as possible, then we might expect the two species to adjust so as to spend the most time possible in a roughly coincident state - an example of adaptive behavior of the two species to maximize their populations. Since species $u$ overtakes species $v$, so that in effect each $v$-island will attach itself to the overtaking $u$-island, one way for this to happen is for the $v$-islands to slow down and assist the $u$-islands in overtaking them. This may explain the nearly vertical behavior of the $v$-contours in Figure 7(b) immediately preceding the re-attachment process. Of course, another way this could happen is for the $u$-islands to speed up, but the maximum speed may be set by the degree of the asymmetry (i.e., $\alpha$ ) and since $\alpha_{u}>\alpha_{v}$ the $u$-islands cannot speed up any further and optimality for both species results if the speed of the $u$-islands stays at this maximum value, hence the straight contours in Figure 7(b). When $\alpha_{v}=0$ (Figure 7(a)) the $v$-islands are already stationary, so they can enhance the process of being overtaken by the $u$-islands by a retrograde motion as can be seen in Figure 7(a).

We next consider the behavior when the coupling coefficients are sufficiently large so that (2.1) can no longer be considered as a small perturbation of the decoupled system. In this case it is reasonable to expect that the increased interspecies coupling would promote a binding together of the waves for the two species. This in fact does happen. In Figure 9 we illustrate the pattern obtained for $\alpha_{u}=0.5$ and $\alpha_{v}=0$ and with $\epsilon$ increased to 0.4 . The two waves now propagate together as bound traveling waves. (We felt this figure came out clearer using a contour level for $u$ sufficiently close to the maximum of $u$ so that the contour levels for $u$ are visible as essentially one line.)

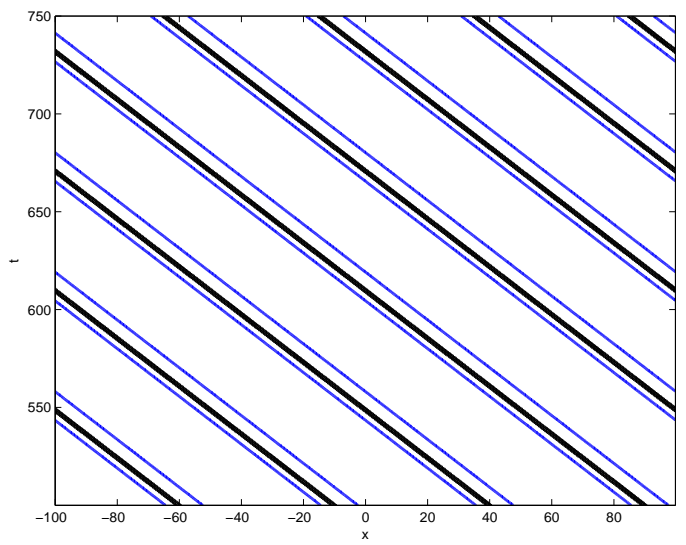

Figure 9. Contour plots of $u$ and $v$ with $\alpha_{u}=0.5$ and $\alpha_{v}=0.0$. Other parameters are given in (4.1) with $\delta=40$ and $\epsilon=0.4$. Kernel function is (2.3). $v$-contour is in blue in online version of this paper.

In order to further clarify the effect of coupling on the binding of the two species we performed a series of computations with $\alpha_{u}=0.5$ and $\alpha_{v}=-0.5$, so that the magnitude of the asymmetry is the same for each species, but is in opposite directions. Furthermore, we modified (4.1) by taking $b_{1}=b_{2}=\epsilon$, 
so that the two species had exactly the same kinetic parameters. For $\epsilon=0.1$ (weak coupling) the two species propagated with the same speed but in opposite directions, with some distortion in the island structure when the waves intersected. In contrast, for $\epsilon=0.8$ (strong coupling) the steady state solution was stationary, i.e., the island/deadzone structure was maintained but the strong coupling together with the opposing asymmetry canceled out any propagation.

\subsection{Asymmetric Gaussian}

We next considered (2.1) with the asymmetric Gaussian (2.4). The parameters were the same as in (4.1) and as above we took $\delta=40$. In this case, if $\alpha_{u}=\alpha_{v}=\alpha$, then the coexistence state with $\alpha=0.5$ was stable, however, patterns formed for $\alpha \geq 0.75$ and random initial conditions led to a two-island propagating array.

In Figure 10(a) contours of $u$ and $v$ are shown for $\alpha_{u}=1$ and $\alpha_{v}=0$. In this case the two species are bound together and propagate as a traveling wave. The $v$-islands are slightly off center with respect to the $u$-islands as shown in Figure 10(b) and we chose the contour levels to accentuate this. We believe that the binding between $u$ and $v$ is a consequence of the fact that for the decoupled problem there is now only one eigenvalue crossing from the left half plane into the right half plane due to the fact that the scalar equation is now unconditionally stable for $\alpha_{v}=0$.

In order to illustrate this, Figure 11(a) shows contours for $\alpha_{u}=1$ and $\alpha_{v}=0.75$. For this case, for the decoupled system there are two eigenvalues crossing from left to right, or said another way, species $v$ left to itself would evolve to its own propagating mode distinct from that of species $u$. When the species are weakly coupled, these two eigenvalues give rise to the unbound waves shown in the figure. We note that for this case the $v$-islands are still off center with regard to the $u$-islands.

In Figure 11(b) we illustrate the case $\alpha_{u}=\alpha_{v}=1$. The two waves are now bound together. In addition, the $u$ and $v$ islands occupy essentially the same space as was also the case for the computations shown in [12] albeit for different parameter values.

As a last example, we show that even when $\alpha_{v}=\alpha_{u}$ unbound waves can occur for sufficiently weak coupling due to two eigenvalues crossing from left to right, one associated with species $u$ and the other with species $v$. We consider the case $\alpha_{u}=\alpha_{v}=1$, with $\epsilon=0.01$. In Figure 12(a) we show contours of $u$ and $v$ over a very long time interval. The figure shows the two species propagating at slightly different speeds. In this case for $\epsilon=0$ the decoupled system has a double eigenvalue crossing from the left to the right and so for $\epsilon=0.01$ the resulting speeds are very close together, necessitating the long time interval used in the figure. (We point out that we carefully checked the speeds for the case in Figure 11(b) $(\epsilon=0.1)$ and found no quantitative difference between them, while a quantitative analysis of the speeds in this case shows a small but definite discrepancy between the wave speeds of the two species.)

Finally, when we changed the kinetic parameters so that for the decoupled system, $\epsilon=0$, the two eigenvalues were different, the divergence of the two waves became significantly more pronounced. Specifically, we considered the parameter set

$$
d_{1}=1, d_{2}=2, c_{1}=1, c_{2}=0.5, a_{1}=a_{2}=1, b_{1}=\epsilon, b_{2}=2 \epsilon, L=100,
$$

with $\epsilon=0.01$. Results are shown in Figure 12(b) where in this case we chose contour levels so that on the scale of the figure each propagating island shows up as essentially one line rather than two. Comparison of this figure with Figure 12(a) (both figures taken over the same time interval) shows more frequent interactions between the propagating island arrays for each species which we infer is due to the spread between the eigenvalues for the decoupled system.

\section{Conclusions and summary}

We have considered the problem of competing species with quadratic nonlinearity, based on a system of nonlocal Fisher-type equations with coupling due to nonlocal species competition. We have investigated the effect of species specific nonlocality on the resulting patterns, focusing on species specific length scales 


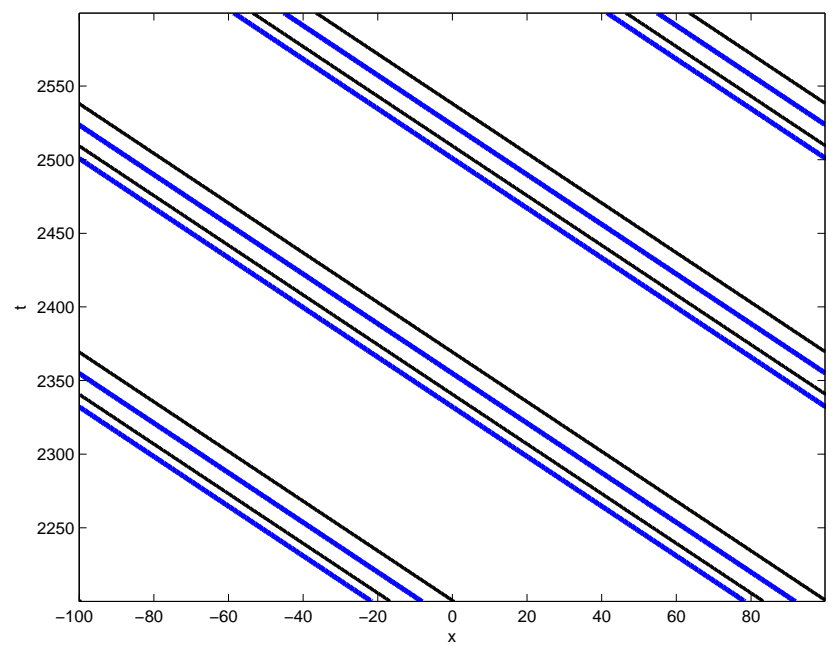

(a) Contour plots of $u$ and $v$

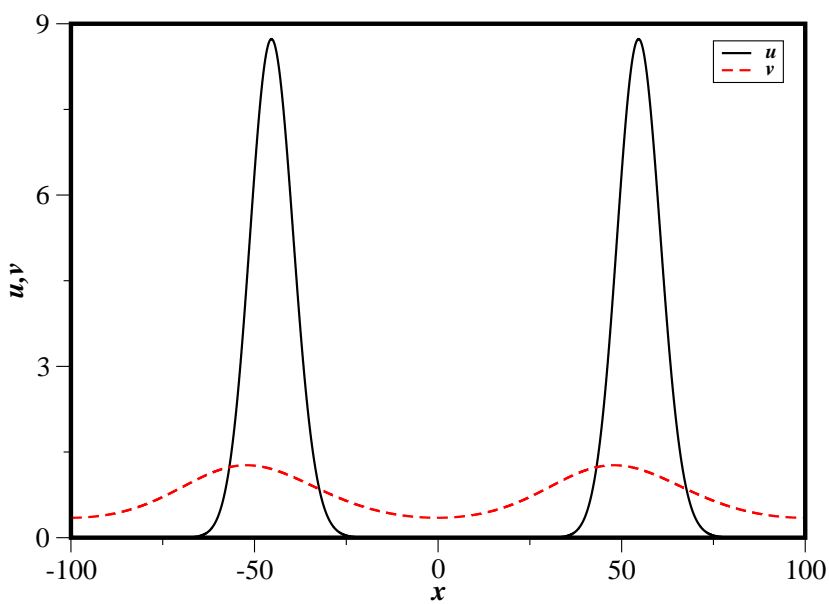

(b) Spatial profiles of $u$ and $v$ at a fixed time.

FiguRE 10. Here $\alpha_{u}=1$ and $\alpha_{v}=0$. Other parameters are given in (4.1) with $\delta=40$ and $\epsilon=0.1$. Kernel function is (2.4).

in the nonlocality and species specific degrees of asymmetry. In both cases we find significantly different patterns from those found when parameters of the nonlocality are the same for both species.

In the case of species specific length scales for the extent of the nonlocality, we assume symmetric coupling for both species and focus on stationary patterns associated with sufficiently large spatial nonlocality specified by a length scale parameter $\delta$. We introduce species specificity via a parameter $C$ so that the length scales for species $u$ and $v$ are $\delta_{u}=\delta$ and $\delta_{v}=\delta / C$, respectively where $C>1$.

For $C \simeq 1$ patterns involve arrays of islands separated by deadzones where the populations are essentially extinct. The islands have roughly the shape of the upper lobe of a cosine curve. This behavior has been observed previously for the case of $C=1$ (length scale the same for both species). However, for $C$ large, the islands for the more local species $v$ take the form of plateaus, at roughly the value of the 


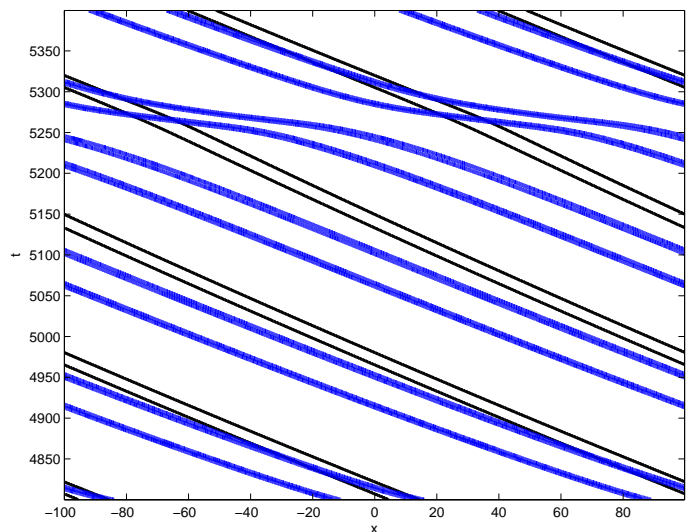

(a) $\alpha_{u}=1, \alpha_{v}=0.75$

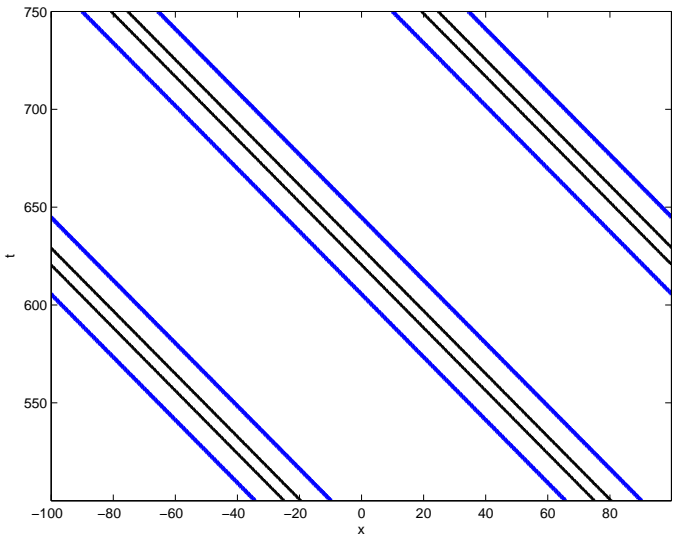

(b) $\alpha_{u}=\alpha_{v}=1$

Figure 11. Contour plots of $u$ and $v$. Parameters are given in (4.1) with $\delta=40$ and $\epsilon=0.1$. Kernel function is (2.4). $v$-contour is in blue in online version of this paper.

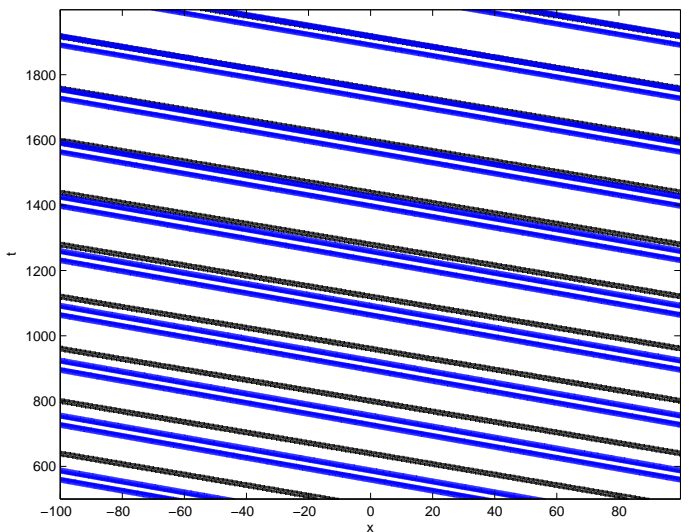

(a) Parameter set (4.1).

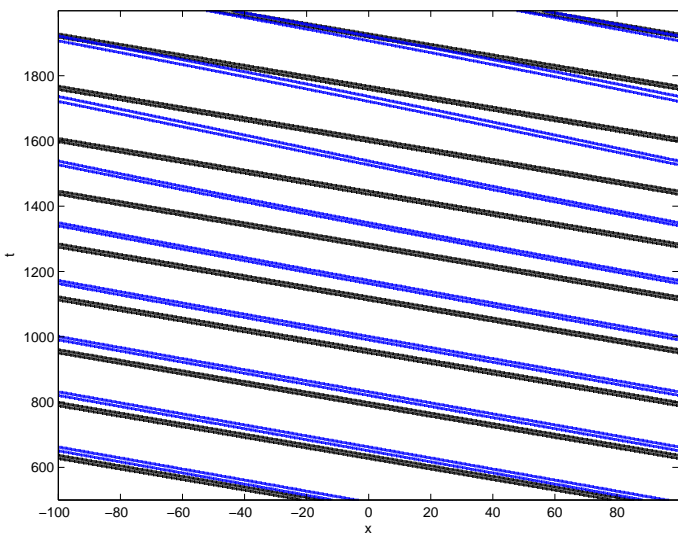

(b) Parameter set (4.2).

Figure 12. Contour plots of $u$ and $v$ with $\alpha_{u}=\alpha_{v}=1, \delta=40$ and $\epsilon=0.01$. Kernel function is (2.4). $v$-contour is in blue in online version of this paper.

$v$-component of the coexistence critical point. This can be attributed to the fact that competition terms involving $v$ are near local, while the competition terms involving $u$ are roughly constant over most of the extent of the $u$-island due to the large value of $\delta_{u}(=\delta)$.

The plateau island structure becomes unstable as $C$ decreases, leading to patterns with stationary oscillations across them. This is essentially a consequence of the instability of the scalar equation for species $v$ with the $u$-contribution to interspecies competition effectively a constant source term in the scalar equation. We showed that the number of maxima across each island is an effective way to categorize the different patterns. As $C$ decreases further split patterns occur where the $u$-island splits into two subislands with a near deadzone between them. This is a consequence of reduced competition from species $v$, due to more of the deadzone being included in the nonlocal competition. Finally for $C$ sufficiently close to unity, additional competition from the adjacent island terminates the split behavior and allows for the formation of intact islands analogous to those seen in [11] for $C=1$. 
For the case of species specific asymmetry, we introduced parameters $\alpha_{u}$ and $\alpha_{v}$ which measured the degree of asymmetry in the nonlocal interaction. Previous studies have considered only the case where $\alpha_{u}=\alpha_{v}=\alpha$, i.e., the asymmetry was the same for both species [12]. For asymmetric nonlocal coupling the patterns are not stationary but rather propagating arrays of islands and deadzones. This is due to instabilities occurring as eigenvalues crossed from the left half plane to the right half plane off the imaginary axis. We concentrated on the behavior associated with the propagating patterns for weakly coupled systems, where for each species the coefficient of the nonlocal interspecies competition term was small. We distinguished two families of patterns, (i) bound patterns where the two species propagated as a bound traveling wave with the same speed and (ii) unbound patterns, where the two species propagated with different speeds, interacting only when the waves intersected, i.e., when both species occupied the same or closely spaced regions.

For weakly coupled systems, the nature of the ensuing patterns, bound or unbound, depended on the behavior of the associated decoupled system. When the individual equations of the decoupled system were unstable, so that each species exhibited its own specific propagation speed (which could be zero if $\alpha_{v}=0$ ) then the waves were unbound. Each species propagated according to the speed it would have for the decoupled equation. This was the case when the kernel function for the nonlocality was a stepfunction. In contrast, when one of the equations for the decoupled system was stable, as was the case when $\alpha_{v}=0$ and the kernel function was a Gaussian, then the two species propagated as a bound wave. The stable species was "slaved" to the unstable species and both species propagated together with the same speed. For all cases which we considered the patterns became bound for sufficiently large coupling parameters. We note that these results may be applicable to other weakly coupled systems, e.g., the coupling need not be nonlocal.

Acknowledgements. This work was supported by NSF RTG grant DMS-0636574.

\section{References}

[1] N. F. Britton. Aggregation and the competitive exclusion principle, J. Theoret. Biol., 136 (1989), 57-66.

[2] N. F. Britton. Spatial structures and periodic travelling waves in an integro-differential reaction-diffusion population model, SIAM. J. Appl. Math., 50 (1990), 1663-1688.

[3] M. A. Fuentes, M. N. Kuperman, V. M. Kenkre. Nonlocal interaction effects on pattern formation in population dynamics, Phys. Rev. Letters, 91 (2003), 158104-1 - 158104-4.

[4] M. A. Fuentes, M. N. Kuperman, V. M. Kenkre. Analytical considerations in the study of spatial patterns arising from nonlocal interaction effects, J. Phys. Chem. B, 108 (2004), 10505-10508.

[5] S. Genieys, N. Bessonov, V. Volpert. Mathematical model of evolutionary branching, Mathematical and Computer Modelling, 49 (2009), 2109-2115.

[6] S. Genieys, V. Volpert, P. Auger. Pattern and waves for a model in population dynamics with nonlocal consumption of resources, Math. Model. Nat. Phenom., 1 (2006), 63-80.

[7] S. Genieys, V. Volpert, P. Auger. Adaptive dynamics: Modelling Darwin's divergence principle, C. R. Biologies, 329 (2006), 876-879.

[8] S. S. Gourley, M. A. J. Chaplain, F. A. Davidson. Spatio-temporal pattern Formation in a nonlocal reaction-diffusion equation, Dynamical Systems, 16 (2001), 173-192.

[9] S. M. Merchant, W. Nagata. Instabilities and spatiotemporal patterns behind predator invasions with nonlocal prey competition, Theoretical Population Biology, 80 (2011), 289-297.

[10] B. Perthame, S. Genieys. Concentration in the nonlocal Fisher equation: the Hamilton-Jacobi limit, Math. Model. Nat. Phenom., 2 (2007), 135-151.

[11] B. L. Segal, V. A. Volpert, A. Bayliss. Pattern formation in a model of competing populations with nonlocal interactions, Physica D, 253 (2013), 12-22.

[12] M. C. Tanzy, V. A. Volpert, A. Bayliss, M. E. Nehrkorn. Stability and pattern formation for competing populations with asymmetric nonlocal coupling, Mathematical Biosciences, 246 (2013), 14-26.

[13] M. C. Tanzy, V. A. Volpert, A. Bayliss, M. E. Nehrkorn, A Nagumo-type model for competing populations with nonlocal coupling, Mathematical Biosciences, 263 (2015), 70-82. 